FACTA UNIVERSITATIS (NIŠ)

Ser. Math. Inform. Vol. 36, No 3 (2021), 557 - 573

https://doi.org/10.22190/FUMI201115041J

Original Scientific Paper

\title{
LINEAR SYSTEMS OF DIFFERENTIAL EQUATIONS IN ARROWHEAD FORM
}

\author{
Ivana Jovović \\ University of Belgrade, School of Electrical Engineering, 11000 Belgrade, Serbia
}

\begin{abstract}
This paper deals with different approaches for solving linear systems of the first order differential equations with the system matrix in the symmetric arrowhead form. Some needed algebraic properties of the symmetric arrowhead matrix are proposed. We investigate the form of invariant factors of the arrowhead matrix. Also the entries of the adjugate matrix of the characteristic matrix of the arrowhead matrix are considered. Some reductions techniques for linear systems of differential equations with the system matrix in the arrowhead form are presented.

Keywords: Arrowhead matrices, Linear systems of differential equations, Partial and total reductions of non-homogeneous linear systems of first order operator equations
\end{abstract}

\section{Introduction}

Arrowhead matrices are an important type of matrices occurring in wide area of applications. They are popular subject of research related with mathematics, physics and engineering. Some important problems like computing eigenvalues and eigenvectors of arrowhead matrices [9, 2, 21], solving inverse eigenvalue problems $[15,28,25,24]$, computing the inverse of arrowhead matrices [7, 26, 4], and solving symmetric arrowhead systems [5] have been considered by various authors over the last four decades. Arrowhead matrices are often an essential tool for the computation of the eigenvalue problems for large and sparse or tridiagonal matrices [22, 6, 8, 29, 23]. Arrowhead matrices arise in the description of modelling of radiationless transitions in isolated molecules [1], oscillators vibrationally coupled with a Fermi liquid [3]

Received November 15, 2020. accepted June 18, 2021.

Communicated by Predrag Stanimirović

Corresponding Author: Ivana Jovović, University of Belgrade, School of Electrical Engineering, 11000 Belgrade, Serbia | E-mail: ivana@etf.bg.ac.rs

2010 Mathematics Subject Classification. Primary 15A21; Secondary 34A30

(C) 2021 by University of Niš, Serbia | Creative Commons License: CC BY-NC-ND 
and quantum optics [19]. One can also find arrowhead matrices in the models of telecommunication systems (MIMO) [27, 14] and neural networks [18], as well as in robotics and in modern control theory. Motivated by wide applications of arrowhead matrices we are interested in solving linear system of differential equations with the system matrix in the symmetric arrowhead form.

In our previous papers we have considered a partial and a total reduction of nonhomogenous linear systems of the first order operator equations with system matrix in an arbitrary form. In [16] the idea was to use the rational canonical form to reduce such a system to an equivalent partially reduced one. The partially reduced system obtained in this fashion consists of higher-order linear operator equations in one variable and first-order linear operator equations in two variables. Another method for solving a linear systems of operator equations, which does not require a change of basis, is discussed in [17]. Obtained totally reduced system consists of higher order operator equations which only differ in the variables and in the non-homogeneous terms. In [12] and [11] we have considered a partial and a total reduction of linear systems of operator equations with the system matrix in the companion form. Papers $[12,11,10]$ and $[13]$ expand our research to non-homogeneous linear systems of operator equations involving more than one operator.

This paper deals with both types of reductions, a partial and a total, of linear systems of the first order differential equations with the system matrix in the arrowhead form. We will look more closely at the form of invariant factors of the arrowhead matrix, which we will use for partial reduction. The adjugate matrix of characteristic matrix of the arrowhead matrix presented as polynomial with matrix coefficients will be used to establish the form for the totally reduced system.

In what follows we propose some important properties of arrowhead matrices, and we will start with definition of the arrowhead matrix.

\section{Some properties of symmetric arrowhead matrices}

A matrix $B \in \mathbb{R}^{n \times n}$ is called a symmetric arrowhead matrix if it has a form

$$
\left[\begin{array}{cccccc}
a_{1} & b_{2} & b_{3} & \ldots & b_{n-1} & b_{n} \\
b_{2} & a_{2} & 0 & \ldots & 0 & 0 \\
b_{3} & 0 & a_{3} & \ldots & 0 & 0 \\
\vdots & \vdots & \vdots & \ddots & \vdots & \vdots \\
b_{n-1} & 0 & 0 & \ldots & a_{n-1} & 0 \\
b_{n} & 0 & 0 & \ldots & 0 & a_{n}
\end{array}\right] .
$$

It is a symmetric matrix obtained by bordering the diagonal matrix with a row and a column with the same elements. The characteristic polynomial of the arrowhead matrix $B$ is

$$
\Delta_{B}(\lambda)=\operatorname{det}(\lambda I-B)=\prod_{i=1}^{n}\left(\lambda-a_{i}\right)-\sum_{i=2}^{n} b_{i}^{2} \prod_{\substack{j=2 \\ j \neq i}}^{n}\left(\lambda-a_{j}\right) .
$$


This formula can be easily derived by expanding the determinant of the matrix $\lambda I-B$ by the first row. The proof of this can be found in [20] and therefore it is omitted here. We denote by $d_{k}, 0 \leq k \leq n$, the coefficient of the term of degree $n-k$ of the characteristic polynomial $\Delta_{B}(\lambda)$. Therefore, we have

$$
\begin{aligned}
& d_{0}=1, \quad d_{1}=-\sum_{i=1}^{n} a_{i}, \quad d_{2}=\sum_{1 \leq i<j \leq n} a_{i} a_{j}-\sum_{i=2}^{n} b_{i}^{2} \text { and }
\end{aligned}
$$

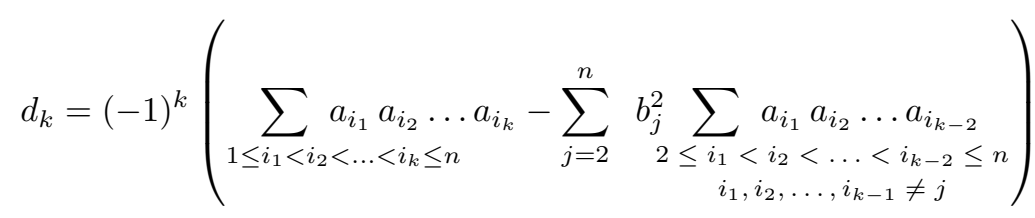

for $3 \leq k \leq n$.

Suppose that $a_{2}>a_{3}>\ldots>a_{n}$ and $b_{i} \neq 0$, for $2 \leq i \leq n$. Then by Cauchy's Interlacing Theorem the eigenvalues $\lambda_{i}$ of the matrix $B, 1 \leq i \leq n$, are distinct. Moreover, if $\lambda_{1}>\lambda_{2}>\ldots>\lambda_{n}$, then $\lambda_{1}>a_{2}>\lambda_{2}>a_{3}>\ldots>a_{n}>\lambda_{n}$. For more details, we refer the reader to [24]. If for some $i, 2 \leq i \leq n, b_{i}=0$, then $a_{i}$ is eigenvalue of the matrix $B$. If the number of repetition of the element $a_{i}$ along the diagonal except on the position $(1,1)$ of the matrix $B$ is $k_{i}$, then the element $a_{i}$ is an eigenvalue of the matrix $B$ with algebraic multiplicity at least $k_{i}-1$. The result follows directly from the equation $(2.2)$, since $\left(\lambda-a_{i}\right)^{k_{i}-1}$ is a factor of $\Delta_{B}(\lambda)$. If the matrix $B$ is of the form

$$
\left[\begin{array}{ccccccccccc}
a_{1} & b_{2} & \ldots & b_{i-1} & b_{i_{1}} & \ldots & b_{i_{k_{i}}} & b_{i+k_{i}} & \ldots & b_{n-1} & b_{n} \\
b_{2} & a_{2} & \ldots & 0 & 0 & \ldots & 0 & 0 & \ldots & 0 & 0 \\
\vdots & \vdots & \ddots & \vdots & \vdots & \ddots & \vdots & \vdots & \ddots & \vdots & \vdots \\
b_{i-1} & 0 & \ldots & a_{i-1} & 0 & \ldots & 0 & 0 & \ldots & 0 & 0 \\
b_{i_{1}} & 0 & \ldots & 0 & a_{i} & \ldots & 0 & 0 & \ldots & 0 & 0 \\
\vdots & \vdots & \ddots & \vdots & \vdots & \ddots & \vdots & \vdots & \ddots & \vdots & \vdots \\
b_{i_{i}} & 0 & \ldots & 0 & 0 & \ldots & a_{i} & 0 & \ldots & 0 & 0 \\
b_{i+k_{i}} & 0 & \ldots & 0 & 0 & \ldots & 0 & a_{i+k_{i}} & \ldots & 0 & 0 \\
\vdots & \vdots & \ddots & \vdots & \vdots & \ddots & \vdots & \vdots & \ddots & \vdots & \vdots \\
b_{n-1} & 0 & \ldots & 0 & 0 & \ldots & 0 & 0 & \ldots & a_{n-1} & 0 \\
b_{n} & 0 & \ldots & 0 & 0 & \ldots & 0 & 0 & \ldots & 0 & a_{n}
\end{array}\right]
$$

for $a_{i} \neq a_{j}, 2 \leq j \leq i-1, i+k_{i} \leq j \leq n$, we will say that elements $b_{i_{1}}, \ldots, b_{i_{k_{i}}}$ correspond to the diagonal element $a_{i}$. According to Corollary 4 in [27] we have 
$\Delta_{B}(\lambda)=\left(\lambda-a_{i}\right)^{k_{i}-1} \Delta_{\widetilde{B}}(\lambda)$, where

$$
\widetilde{B}=\left[\begin{array}{ccccccccc}
a_{1} & b_{2} & \ldots & b_{i-1} & \sqrt{\sum_{j=1}^{k_{i}} b_{i_{j}}^{2}} & b_{i+k_{i}} & \ldots & b_{n-1} & b_{n} \\
b_{2} & a_{2} & \ldots & 0 & 0 & 0 & \ldots & 0 & 0 \\
\vdots & \vdots & \ddots & \vdots & \vdots & \vdots & \ddots & \vdots & \vdots \\
b_{i-1} & 0 & \ldots & a_{i-1} & 0 & 0 & \ldots & 0 & 0 \\
\sqrt{\sum_{j=1}^{k_{i} b_{i_{j}}^{2}}} & 0 & \ldots & 0 & a_{i} & 0 & \ldots & 0 & 0 \\
b_{i+k_{i}} & 0 & \ldots & 0 & 0 & a_{i+k_{i}} & \ldots & 0 & 0 \\
\vdots & \vdots & \ddots & \vdots & \vdots & \vdots & \ddots & \vdots & \vdots \\
b_{n-1} & 0 & \ldots & 0 & 0 & 0 & \ldots & a_{n-1} & 0 \\
b_{n} & 0 & \ldots & 0 & 0 & 0 & \ldots & 0 & a_{n}
\end{array}\right]
$$

Characteristic polynomial of the matrix $\widetilde{B}$ is polynomial

$$
\Delta_{\widetilde{B}}(\lambda)=\prod_{j=1}^{i}\left(\lambda-a_{j}\right) \prod_{j=i+k_{i}}^{n}\left(\lambda-a_{j}\right)-\sum_{j=2}^{n+1-k_{i}} \widetilde{b}_{j}^{2} \prod_{\substack{k=2 \\ k \neq j}}^{i}\left(\lambda-a_{k}\right) \prod_{\substack{k=i+k_{i} \\ k \neq j}}^{n}\left(\lambda-a_{k}\right)
$$

where $\widetilde{b}_{j}=b_{j}$ for $2 \leq j \leq i-1, \widetilde{b}_{i}=\sqrt{\sum_{j=1}^{k_{i}} b_{i_{j}}^{2}}$ and $\widetilde{b}_{j-k_{i}+1}=b_{j}$ for $i+k_{i} \leq j \leq n$. We would like to investigate under what condition $a_{i}$ is an eigenvalue of the matrix $\widetilde{B}$. We have $\Delta_{\widetilde{B}}\left(a_{i}\right)=-\widetilde{b}_{i}^{2} \prod_{k=2}^{i-1}\left(a_{i}-a_{k}\right) \prod_{k=i+k_{i}}^{n}\left(a_{i}-a_{k}\right)$, and since $a_{i} \neq a_{j}$ for $2 \leq j \leq i-1$ and $i+k_{i} \leq j \leq n$ we deduce that $a_{i}$ is an eigenvalue of $\widetilde{B}$ if and only if $\widetilde{b}_{i}^{2}=\sqrt{\sum_{j=1}^{k_{i}} b_{i_{j}}^{2}}=0$, i.e., if and only if $b_{i_{j}}=0$ for all $j, 1 \leq j \leq k_{i}$. Therefore, $a_{i}$ is an eigenvalue of $\widetilde{B}$ if and only if all corresponding elements to the diagonal element $a_{i}$ in $B$ are zeros. So, in this case algebraic multiplicity of the element $a_{i}$ in the matrix $B$ is $k_{i}$. If there is at least one non-zero corresponding element to $a_{i}$, algebraic multiplicity of $a_{i}$ is $k_{i}-1$. Let $a_{i_{1}}, a_{i_{2}}, \ldots, a_{i_{p}}$ be different elements along the diagonal with corresponding elements all equal to zero and let $a_{j_{1}}, a_{j_{2}}, \ldots, a_{j_{q}}$ be different elements along the diagonal with at least one corresponding element different from zero. Let $k_{i_{t}}$ and $k_{j_{s}}, 1 \leq t \leq p, 1 \leq s \leq q$, be the numbers of repetition of the elements $a_{i_{t}}$ and $a_{j_{s}}$ along the diagonal except on the position $(1,1)$ and define $m_{j_{s}}$ by $m_{j_{s}}=\left\{\begin{array}{ll}1, & k_{j_{s}}>1 \\ 0, & k_{j_{s}}=1 .\end{array}\right.$ Then the minimal polynomial of the matrix $B$ is of the form

$$
\mu_{B}(\lambda)=\prod_{s=1}^{p}\left(\lambda-a_{i_{s}}\right) \prod_{s=1}^{q}\left(\lambda-a_{j_{s}}\right)^{m_{j_{s}}} \Delta_{\widetilde{B}}(\lambda)
$$


where $\widetilde{B}$ is completely reduced arrowhead matrix, i.e., it is a matrix of the form

$$
\left[\begin{array}{cccc}
a_{1} & \widetilde{b}_{j_{1}} & \ldots & \widetilde{b}_{j_{q}} \\
\widetilde{b}_{j_{1}} & a_{j_{1}} & \ldots & 0 \\
\vdots & \vdots & \ddots & \vdots \\
\widetilde{b}_{j_{q}} & 0 & \ldots & a_{j_{q}}
\end{array}\right]
$$

$\widetilde{b}_{j_{s}}=\sqrt{\sum_{t=1}^{k_{s_{s}}} b_{j_{s_{t}}}^{2}}$ and $b_{j_{s_{t}}}$ are corresponding elements of the element $a_{j_{s}}$ in the matrix $B, 1 \leq s \leq q$. If the elements $a_{2}, a_{3}, \ldots, a_{n}$ are all different, then the minimal and characteristic polynomials of the matrix $B$ are the same. Otherwise, since matrix $B$ is symmetric the number of its invariant factors is equal to $k=$ $\max \left\{k_{i_{1}}, \ldots, k_{i_{p}}, k_{j_{1}}-1, \ldots, k_{j_{q}}-1\right\}$. The $k$-th invariant factor of the matrix $B$ is $\mu_{B}(\lambda)$. The $m$-th invariant factor of the matrix $B, 1 \leq m \leq k-1$ is the polynomial

$$
\tau_{m}(\lambda)=\prod_{s=1}^{p}\left(\lambda-a_{i_{s}}\right)^{g_{i_{s}}} \prod_{s=1}^{q}\left(\lambda-a_{j_{s}}\right)^{g_{j_{s}}}
$$

where $g_{i_{s}}=\left\{\begin{array}{ll}1, & k_{i_{s}}-(k-m)>0 \\ 0, & \text { otherwise }\end{array}\right.$ and $g_{j_{s}}= \begin{cases}1, & k_{j_{s}}-(k-m)>1 \\ 0, & \text { otherwise. }\end{cases}$

From now on we will be concern with the coefficients of the adjugate matrix of the characteristic matrix of the symmetric arrowhead matrix $B$. Suppose that the adjugate matrix of the characteristic matrix $\lambda I-B$ is written in the form

$$
\operatorname{adj}(\lambda \mathrm{I}-\mathrm{B})=\lambda^{\mathrm{n}-1} \mathrm{~B}_{0}+\lambda^{\mathrm{n}-2} \mathrm{~B}_{1}+\ldots+\lambda \mathrm{B}_{\mathrm{n}-2}+\mathrm{B}_{\mathrm{n}-1} .
$$

Let us determine the coefficients $B_{k}$ using recurrences $B_{k}=B \cdot B_{k-1}+d_{k} I$, for $1 \leq k \leq n-1$, and $B_{0}=I$. The recurrences are obtained by equating coefficients at the same powers of $\lambda$ on both sides of the equality adj $(\lambda \mathrm{I}-\mathrm{B}) \cdot(\lambda \mathrm{I}-\mathrm{B})=\Delta_{\mathrm{B}}(\lambda) \mathrm{I}$.

Lemma 2.1. The coefficient $B_{k}=\left[b_{i j}^{k}\right]_{n \times n}, 2 \leq k \leq n-1$, of the matrix $\operatorname{adj}(\lambda \mathrm{I}-\mathrm{B})$ is matrix with entries

$$
\begin{aligned}
& b_{11}^{k}=(-1)^{k} \sum a_{i_{1}} a_{i_{2}} \ldots a_{i_{k}} \quad b_{1 j}^{k}=(-1)^{k-1} b_{j} \sum a_{i_{1}} a_{i_{2}} \ldots a_{i_{k-1}} \\
& 2 \leq i_{1}<i_{2}<\ldots<i_{k} \leq n \quad 2 \leq i_{1}<i_{2}<\ldots<i_{k-1} \leq n \\
& i_{1}, i_{2}, \ldots, i_{k-1} \neq j \\
& b_{j 1}^{k}=(-1)^{k-1} b_{j} \sum a_{i_{1}} a_{i_{2}} \ldots a_{i_{k-1}} \quad b_{i j}^{k}=(-1)^{k-2} b_{i} b_{j} \sum a_{i_{1}} a_{i_{2}} \ldots a_{i_{k-2}} \\
& \begin{array}{cc}
2 \leq i_{1}<i_{2}<\ldots<i_{k-1} \leq n & 2 \leq i_{1}<i_{2}<\ldots<i_{k-2} \leq n \\
i_{1}, i_{2}, \ldots, i_{k-1} \neq j & \leq n \\
i_{1}, i_{2}, \ldots, i_{k-2} \neq i, j &
\end{array}
\end{aligned}
$$

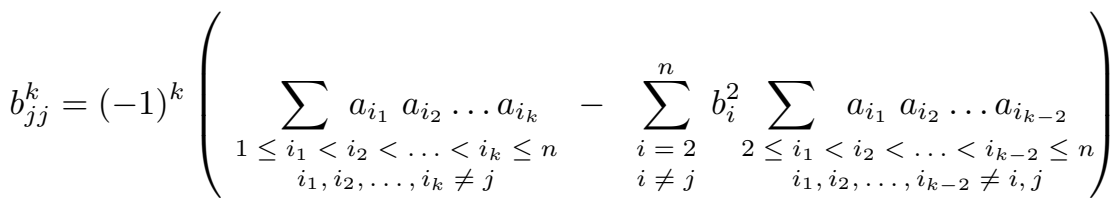

for $2 \leq i, j \leq n$ and $i \neq j$. 
Proof. The proof proceeds by induction on $k$. We have $B_{0}=I$. For coefficient $B_{1}$ holds $B_{1}=B \cdot I+d_{1} I$, i.e.,

$$
B_{1}=\left[\begin{array}{ccccc}
-\sum_{i=2}^{n} a_{i} & b_{2} & \ldots & b_{n-1} & b_{n} \\
b_{2} & -\sum_{i=1, i \neq 2}^{n} a_{i} & \ldots & 0 & 0 \\
\vdots & \vdots & \ddots & \vdots & \vdots \\
b_{n-1} & 0 & \ldots & -\sum_{i=1, \neq n-1}^{n} a_{i} & 0 \\
b_{n} & 0 & \ldots & 0 & -\sum_{i=1}^{n-1} a_{i}
\end{array}\right] .
$$

Coefficient $B_{1}$ is also arrowhead matrix. Let $(B)_{\rightarrow j}$ stand for the $j$-th row of the matrix $B$, and let $\left(B_{k-1}\right)_{\downarrow j}$ denote the $j$-th column of the matrix $B_{k-1}, 1 \leq j \leq n$. Assume that coefficients of the matrix $B_{k-1}$ satisfy required form. Then we have

$$
\begin{aligned}
& b_{11}^{k}=(B)_{\rightarrow 1} \cdot\left(B_{k-1}\right)_{\downarrow 1}+d_{k}=a_{1} b_{11}^{k-1}+\sum_{j=2}^{n} b_{j} b_{j 1}^{k-1}+d_{k} \\
& =(-1)^{k-1} a_{1} \sum_{2 \leq i_{1}<i_{2}<\ldots<i_{k-1} \leq n} a_{i_{1}} a_{i_{2}} \ldots a_{i_{k-1}}+(-1)^{k-2} \sum_{j=2}^{n} b_{j}^{2} \sum_{2 \leq i_{1}<i_{2}<\ldots<i_{k-2} \leq n} a_{i_{1}} a_{i_{2}} \ldots a_{i_{k-2}} \\
& +(-1)^{k} \sum_{1 \leq i_{1}<i_{2}<\ldots<i_{k} \leq n} a_{i_{1}} a_{i_{2}} \ldots a_{i_{k}}+(-1)^{k-1} \sum_{j=2}^{n} b_{j}^{2} \sum_{2 \leq i_{1}<i_{2}<\ldots<i_{k-2} \leq n} a_{i_{1}} a_{i_{2}} \ldots a_{i_{k-2}} \\
& =(-1)^{k} \sum a_{i_{1}} a_{i_{2}} \ldots a_{i_{k}} \\
& i_{1}, i_{2}, \ldots, i_{k-2} \neq j \\
& 2 \leq i_{1}<i_{2}<\ldots<i_{k} \leq n \\
& b_{j 1}^{k}=(B)_{\rightarrow j} \cdot\left(B_{k-1}\right)_{\downarrow 1}=b_{j} b_{11}^{k-1}+a_{j} b_{j 1}^{k-1} \\
& =(-1)^{k-1} b_{j} \sum_{2 \leq i_{1}<i_{2}<\ldots<i_{k-1} \leq n} a_{i_{1}} a_{i_{2}} \ldots a_{i_{k-1}}+(-1)^{k-2} a_{j} b_{j} \sum_{2 \leq i_{1}<i_{2}<\ldots<i_{k-2} \leq n} a_{i_{1}} a_{i_{2}} \ldots a_{i_{k-2}} \\
& =(-1)^{k-1} b_{j} \sum_{2 \leq i_{1}<i_{2}<\ldots<i_{k-1} \leq n} a_{i_{1}} a_{i_{2}} \ldots a_{i_{k-1}} \\
& i_{1}, i_{2}, \ldots, i_{k-1} \neq j \\
& b_{i j}^{k}=(B)_{\rightarrow i} \cdot\left(B_{k-1}\right)_{\downarrow j}=b_{i} b_{1 j}^{k-1}+a_{i} b_{i j}^{k-1} \\
& =(-1)^{k-2} b_{i} b_{j} \sum_{2 \leq i_{1}<i_{2}<\ldots<i_{k-2} \leq n} a_{i_{1}} a_{i_{2}} \ldots a_{i_{k-2}}+(-1)^{k-3} a_{i} b_{i} b_{j} \sum_{2 \leq i_{1}<i_{2}<\ldots<i_{k-3} \leq n} a_{i_{1}} a_{i_{2}} \ldots a_{i_{k-3}} \\
& i_{1}, i_{2}, \ldots, i_{k-2} \neq j \quad i_{1}, i_{2}, \ldots, i_{k-3} \neq i, j \\
& =(-1)^{k-2} b_{i} b_{j} \sum_{2 \leq i_{1}<i_{2}<\ldots<i_{k-2} \leq n} a_{i_{1}} a_{i_{2}} \ldots a_{i_{k-2}} \\
& i_{1}, i_{2}, \ldots, i_{k-2} \neq i, j
\end{aligned}
$$




$$
\begin{aligned}
& b_{1 j}^{k}=(B)_{\rightarrow 1} \cdot\left(B_{k-1}\right)_{\downarrow j}=a_{1} b_{1 j}^{k-1}+\sum_{\substack{i=2 \\
i \neq j}}^{n} b_{i} b_{i j}^{k-1}+b_{j} b_{j j}^{k-1} \\
& =(-1)^{k-2} a_{1} b_{j} \sum_{\substack{i_{1}<i_{2}<\ldots<i_{k-2} \leq n \\
i_{1}, i_{2}, \ldots, i_{k-2} \neq j}} a_{i_{1}} a_{i_{2}} \ldots a_{i_{k-2}}+(-1)^{k-3} \sum_{\substack{i=2 \\
i \neq j}}^{n} b_{i}^{2} b_{j} \sum_{\substack{i \neq i_{1}<i_{2}<\ldots<i_{k-3} \leq n \\
i_{1}, i_{2}, \ldots, i_{k-3} \neq i, j}} a_{i_{1}} a_{i_{2}} \ldots a_{i_{k-3}} \\
& +(-1)^{k-1} b_{j} \sum_{\substack{i_{1}<i_{2}<\ldots<i_{k-1} \leq n \\
i_{1}, i_{2}, \ldots, i_{k-1} \neq j}} a_{i_{1}} a_{i_{2}} \ldots a_{i_{k-1}}+(-1)^{k} \sum_{\substack{i=2 \\
i \neq j}}^{n} b_{i}^{2} b_{j} \sum_{\substack{i i_{1}<i_{2}<\ldots<i_{k-3} \leq n \\
i_{1}, i_{2}, \ldots, i_{k-3} \neq i, j}} a_{i_{1}} a_{i_{2}} \ldots a_{i_{k-3}} \\
& =(-1)^{k-1} b_{j} \sum_{2 \leq i_{1}<i_{2}<\ldots<i_{k-1} \leq} a_{i_{1}} a_{i_{2}} \ldots a_{i_{k-1}} \\
& \leq i_{1}<i_{2}<\ldots<i_{k-1} \leq n \\
& b_{j j}^{k}=(B)_{\rightarrow j} \cdot\left(B_{k-1}\right)_{\downarrow j}+d_{k}=b_{j} b_{1 j}^{k-1}+a_{j} b_{j j}^{k-1}+d_{k} \\
& =(-1)^{k-2} b_{j}^{2} \sum_{\substack{i_{1}<i_{2}<\ldots<i_{k-2} \leq n \\
i_{1}, i_{2}, \ldots, i_{k-2} \neq j}} a_{i_{1}} a_{i_{2}} \ldots a_{i_{k-2}}+(-1)^{k-1} a_{j} \sum_{\substack{i_{1}<i_{2}<\ldots<i_{k-1} \leq n \\
i_{1}, i_{2}, \ldots, i_{k-1} \neq j}} a_{i_{1}} a_{i_{2}} \ldots a_{i_{k-1}} \\
& +(-1)^{k-2} \sum_{\substack{i=2 \\
i \neq j}}^{n} b_{i}^{2} a_{j} \sum_{\substack{i_{1}<i_{2}<\ldots<i_{k-3} \leq n \\
i_{1}, i_{2}, \ldots, i_{k-3} \neq i, j}} a_{i_{1}} a_{i_{2}} \ldots a_{i_{k-3}}+(-1)^{k} \sum_{1 \leq i_{1}<i_{2}<\ldots<i_{k} \leq n} a_{i_{1}} a_{i_{2}} \ldots a_{i_{k}} \\
& +(-1)^{k-1} \sum_{i=2}^{n} b_{i}^{2} \sum_{2 \leq i_{1}<i_{2}<\ldots<i_{k-2} \leq n} a_{i_{1}} a_{i_{2}} \ldots a_{i_{k-2}} \\
& =(-1)^{k-1} \sum_{\substack{i=2 \\
i \neq j}}^{n} b_{i}^{2} \sum_{\substack{i_{1}<i_{2}<\ldots<i_{k-2} \leq n \\
i_{1}, i_{2}, \ldots, i_{k-2} \neq i}} a_{i_{1}} a_{i_{2}} \ldots a_{i_{k-2}}+(-1)^{k} \sum_{\substack{1 \leq i_{1}<i_{2}<\ldots<i_{k} \leq n \\
i_{1}, i_{2}, \ldots, i_{k} \neq j}} a_{i_{1}} a_{i_{2}} \ldots a_{i_{k}} \\
& +(-1)^{k-2} \sum_{\substack{i=2 \\
i \neq j}}^{n} b_{i}^{2} a_{j} \sum_{\substack{i_{1}<i_{2}<\ldots<i_{k-3} \leq n \\
i_{1}, i_{2}, \ldots, i_{k-3} \neq i, j}} a_{i_{1}} a_{i_{2}} \ldots a_{i_{k-3}} \\
& =(-1)^{k-1} \sum_{\substack{i=2 \\
i \neq j}}^{n} b_{i}^{2} \sum_{\substack{i_{1}<i_{2}<\ldots<i_{k-2} \leq n \\
i_{1}, i_{2}, \ldots, i_{k-2} \neq i, j}} a_{i_{1}} a_{i_{2}} \ldots a_{i_{k-2}}+(-1)^{k} \sum_{\substack{i_{1}<i_{2}<\ldots<i_{k} \leq n \\
i_{1}, i_{2}, \ldots, i_{k} \neq j}} a_{i_{1}} a_{i_{2}} \ldots a_{i_{k}}
\end{aligned}
$$

Therefore, we have shown that coefficients of the matrix $B_{k}$ are of the required form.

\section{The reduction formulas for linear systems of differential equations with the system matrix in the arrowhead form}

Let $C^{\infty}(\mathbb{R})$ be a vector space of all infinitely differentiable functions and let $D: C^{\infty}(\mathbb{R}) \rightarrow C^{\infty}(\mathbb{R})$ be a differential operator on the vector space $C^{\infty}(\mathbb{R})$. We will 
consider non-homogeneous linear system of differential equations with the system matrix in the symmetric arrowhead form

$$
\begin{aligned}
& D\left(x_{1}\right)=a_{1} x_{1}+b_{2} x_{2}+b_{3} x_{3}+\ldots+b_{n-1} x_{n-1}+b_{n} x_{n}+\varphi_{1} \\
& D\left(x_{2}\right)=b_{2} x_{1}+a_{2} x_{2}+\varphi_{2} \\
& D\left(x_{3}\right)=b_{3} x_{1}+a_{3} x_{3}+\varphi_{3} \\
& \vdots \\
& D\left(x_{n-1}\right)=b_{n-1} x_{1}+a_{n-1} x_{n-1}+\varphi_{n-1} \\
& D\left(x_{n}\right)=b_{n} x_{1}+a_{n} x_{n}+\varphi_{n},
\end{aligned}
$$

for $a_{i}, b_{i} \in \mathbb{R}, \varphi_{i} \in C^{\infty}(\mathbb{R}), 1 \leq i \leq n$.

Since symmetric arrowhead matrix is diagonalizable, we can find a general solution of our system by rewriting it in a basis formed by eigenvectors. The obtained system is completely decoupled, so we get a system of $n$ linear differential equations of the first order in one variable. This method is very convenient theoretically, but in actual calculations usually requires quite a few steps. Furthermore, while there are some approaches for finding eigenvalues and eigenvectors of arrowhead matrix it can be a difficult job.

Applying Theorem 3.7 from the paper [16] and taking into consideration the form of the invariant factors of the symmetric arrowhead matrix we obtain the partially reduced system. Partially reduced system consists of $k$ subsystems, where $k$ is a number of invariant factors of system matrix. Every subsystem corresponds to one invariant factor. The first equation of subsystems is non-homogeneous linear differential equation in one unknown, with the characteristic polynomial equal to the invariant factor and with the non-homogenous term equal to the sum of principal minors of some doubly companion matrices obtained by replacing the first column of the companion matrix of the invariant factor by a column of the first and higher order derivatives of non-homogeneous terms involved in subsystem. Remaining equations are linear differential equations of the first order in two variables. This method also requires the change of basis.

The simple form of our system matrix inspire us to try to derive partial reduction formulas directly. In this manner we state following theorem, a direct method for transforming system (3.1) into partially reduced system.

Theorem 3.1. The linear system of the first order differential equations (3.1) can 
be transformed into the partially reduced system

$$
\begin{array}{ll}
\Delta_{B}(D)\left(x_{1}\right) & =\prod_{j=2}^{n}\left(D-a_{j}\right)\left(\varphi_{1}\right)+\sum_{i=2}^{n} b_{i} \prod_{\substack{j=2 \\
j \neq i}}^{n}\left(D-a_{j}\right)\left(\varphi_{i}\right) \\
\left(D-a_{2}\right)\left(x_{2}\right) & =b_{2} x_{1}+\varphi_{2} \\
\left(D-a_{3}\right)\left(x_{3}\right) & =b_{3} x_{1}+\varphi_{3} \\
\vdots & \\
\left(D-a_{n-1}\right)\left(x_{n-1}\right) & =b_{n-1} x_{1}+\varphi_{n-1} \\
\left(D-a_{n}\right)\left(x_{n}\right) & =b_{n} x_{1}+\varphi_{n},
\end{array}
$$

where the linear operator $\Delta_{B}(D)$ is define by replacing $\lambda$ by $D$ in (2.2).

Proof. Let us denote by $\prod_{i=2}^{n}\left(D-a_{i}\right)$ composition of operators $D-a_{i}$, for $2 \leq i \leq n$. The partially reduced system (3.2) is obtained by acting $\prod_{i=2}^{n}\left(D-a_{i}\right)$ on the first equation of the system (3.1) and by substituting expressions $\left(D-a_{i}\right)\left(x_{i}\right)$ appearing on the right sides of equality with $b_{i} x_{1}+\varphi_{i}$, for $2 \leq i \leq n$. Mind that operators $D-a_{i}$ and $D-a_{j}$ commute, for every $i$ and $j$ such that $2 \leq i, j \leq n$. Thus we have

$$
\begin{aligned}
\prod_{j=1}^{n}\left(D-a_{j}\right)\left(x_{1}\right) & =\sum_{i=2}^{n} b_{i} \prod_{j=2}^{n}\left(D-a_{j}\right)\left(x_{i}\right)+\prod_{j=2}^{n}\left(D-a_{j}\right)\left(\varphi_{1}\right)= \\
& =\sum_{i=2}^{n} b_{i} \prod_{\substack{j=2 \\
j \neq i}}^{n}\left(D-a_{j}\right)\left(b_{i} x_{1}+\varphi_{i}\right)+\prod_{j=2}^{n}\left(D-a_{j}\right)\left(\varphi_{1}\right)= \\
& =\sum_{i=2}^{n} b_{i}^{2} \prod_{\substack{j=2 \\
j \neq i}}^{n}\left(D-a_{j}\right)\left(x_{1}\right) \\
& +\sum_{i=2}^{n} b_{i} \prod_{\substack{j=2 \\
j \neq i}}^{n}\left(D-a_{j}\right)\left(\varphi_{i}\right)+\prod_{j=2}^{n}\left(D-a_{j}\right)\left(\varphi_{1}\right) .
\end{aligned}
$$

Rearranging the equation, we get the first equation from (3.2), i.e., we obtain

$$
\Delta_{B}(D)\left(x_{1}\right)=\prod_{j=2}^{n}\left(D-a_{j}\right)\left(\varphi_{1}\right)+\sum_{i=2}^{n} b_{i} \prod_{\substack{j=2 \\ j \neq i}}^{n}\left(D-a_{j}\right)\left(\varphi_{i}\right) .
$$

Finally we are considering total reduction of our arrowhead form system. As an immediate consequence of Theorems 4.1 from the paper [17] we can transform the 
system (3.1) into the totally reduced system

$$
\begin{aligned}
& \Delta_{B}(D)\left(x_{1}\right)=\sum_{k=1}^{n} \sum_{j=1}^{n} b_{1 j}^{(k)} D^{n-k}\left(\varphi_{j}\right) \\
& \Delta_{B}(D)\left(x_{2}\right)=\sum_{k=1}^{n} \sum_{j=1}^{n} b_{2 j}^{(k)} D^{n-k}\left(\varphi_{j}\right) \\
& \vdots \\
& \Delta_{B}(D)\left(x_{n}\right)=\sum_{k=1}^{n} \sum_{j=1}^{n} b_{n j}^{(k)} D^{n-k}\left(\varphi_{j}\right),
\end{aligned}
$$

where the linear operator $\Delta_{B}(D)$ is define by replacing $\lambda$ by $D$ in (2.2) and coefficients $b_{i j}^{(k)}$ are calculated in Lemma 2.1 .

\section{An example}

We will illustrate the previous results by the example. Consider the system of the differential equations

$$
\begin{aligned}
& D\left(x_{1}\right)=x_{1}+x_{2}+2 x_{3}+2 x_{4}+18 e^{t} \\
& D\left(x_{2}\right)=x_{1}+x_{2} \\
& D\left(x_{3}\right)=2 x_{1}+x_{3} \\
& D\left(x_{4}\right)=2 x_{1}+x_{4} .
\end{aligned}
$$

The vector form of the system (4.1) is $D(\vec{x})=B \vec{x}+\vec{\varphi}$, where $\vec{x}=\left[\begin{array}{lll}x_{1} & x_{2} & x_{3} \\ x_{4}\end{array}\right]^{T}$ and

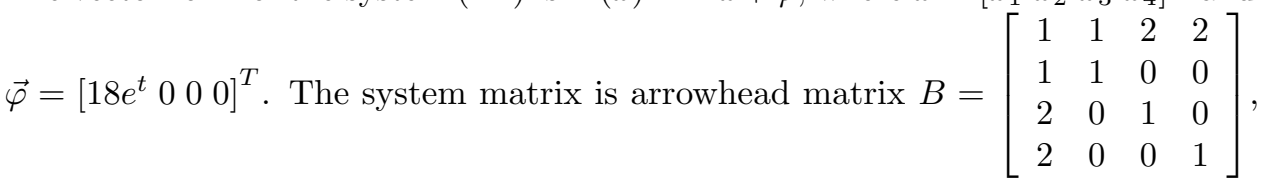
the reduced form of the matrix $B$ is $\widetilde{B}=\left[\begin{array}{ll}1 & 3 \\ 3 & 1\end{array}\right]$, the characteristic polynomial of the matrix $\widetilde{B}$ is $\Delta_{\widetilde{B}}(\lambda)=\left|\begin{array}{ll}\lambda-1 & 3 \\ 3 & \lambda-1\end{array}\right|=(\lambda-1)^{2}-9=(\lambda-4)(\lambda+2)$, and for the characteristic polynomial of the matrix $B$

$$
\Delta_{B}(\lambda)=(\lambda-1)^{2} \Delta_{\widetilde{B}}(\lambda)=(\lambda-1)^{2}(\lambda-4)(\lambda+2)=\lambda^{4}-4 \lambda^{3}-3 \lambda^{2}+14 \lambda-8
$$

holds. Coefficients of the characteristic polynomial of the matrix $B$ are $d_{0}=1$, $d_{1}=-4, d_{2}=-3, d_{3}=14$ and $d_{4}=-8$. The eigenvalues of the matrix $B$ are $\lambda_{1}=\lambda_{2}=1, \lambda_{3}=-2$ and $\lambda_{4}=4$. Corresponding eigenvectors are $v_{1}=\left[\begin{array}{llll}0 & -2 & 1 & 0\end{array}\right]^{T}$, $v_{2}=\left[\begin{array}{llll}0 & -2 & 0 & 1\end{array}\right]^{T}, v_{3}=\left[\begin{array}{llll}-3 & 1 & 2 & 2\end{array}\right]^{T}$ and $v_{4}=\left[\begin{array}{llll}3 & 1 & 2 & 2\end{array}\right]^{T}$. The Jordan normal 
form of the matrix $B$ is $J=\left[\begin{array}{cccc}1 & 0 & 0 & 0 \\ 0 & 1 & 0 & 0 \\ 0 & 0 & -2 & 0 \\ 0 & 0 & 0 & 4\end{array}\right]$, the transformation matrix is $P=$ $\left[\begin{array}{rrrr}0 & 0 & -3 & 3 \\ -2 & -2 & 1 & 1 \\ 1 & 0 & 2 & 2 \\ 0 & 1 & 2 & 2\end{array}\right]$ and its inverse is matrix $P^{-1}=\frac{1}{18}\left[\begin{array}{rrrr}0 & -4 & 10 & -8 \\ 0 & -4 & -8 & 10 \\ -3 & 1 & 2 & 2 \\ 3 & 1 & 2 & 2\end{array}\right]$.

The system (4.1) can be transformed to equivalent system $D(\vec{y})=J \vec{y}+\vec{\psi}$, where $\vec{y}=\left[\begin{array}{llll}y_{1} & y_{2} & y_{3} & y_{4}\end{array}\right]^{T}=P^{-1} \vec{x}$ and $\vec{\psi}=P^{-1} \vec{\varphi}$, i.e., we have

$$
\begin{aligned}
& D\left(y_{1}\right)=y_{1} \\
& D\left(y_{2}\right)=y_{2} \\
& D\left(y_{3}\right)=-2 y_{3}-3 e^{t} \\
& D\left(y_{4}\right)=4 y_{4}+3 e^{t} \text {. }
\end{aligned}
$$

Solution of the previous system is

$$
\left[\begin{array}{l}
y_{1} \\
y_{2} \\
y_{3} \\
y_{4}
\end{array}\right]=\left[\begin{array}{l}
C_{1} e^{t} \\
C_{2} e^{t} \\
C_{3} e^{-2 t}-e^{t} \\
C_{4} e^{4 t}-e^{t}
\end{array}\right]
$$

and the solution of the system (4.1) is

$$
\left[\begin{array}{l}
x_{1} \\
x_{2} \\
x_{3} \\
x_{4}
\end{array}\right]=\left[\begin{array}{l}
-3 C_{3} e^{-2 t}+3 C_{4} e^{4 t} \\
-2\left(C_{1}+C_{2}+1\right) e^{t}+C_{3} e^{-2 t}+C_{4} e^{4 t} \\
\left(C_{1}-4\right) e^{t}+2 C_{3} e^{-2 t}+2 C_{4} e^{4 t} \\
\left(C_{2}-4\right) e^{t}+2 C_{3} e^{-2 t}+2 C_{4} e^{4 t}
\end{array}\right]
$$

The arrowhead matrix $B$ has two invariant factors $\tau_{1}(\lambda)=\lambda-1$ and $\tau_{2}(\lambda)=$ $\mu_{B}(\lambda)=(\lambda-1)(\lambda+2)(\lambda-4)=\lambda^{3}-3 \lambda^{2}-6 \lambda+8$. The rational normal form of the matrix $B$ is $C=\left[\begin{array}{cccc}1 & 0 & 0 & 0 \\ 0 & 0 & 1 & 0 \\ 0 & 0 & 0 & 1 \\ 0 & -8 & 6 & 3\end{array}\right]$, the transformation matrix is $T=$ $\frac{1}{9}\left[\begin{array}{rrrr}0 & -3 & 6 & -3 \\ -18 & -13 & -7 & 2 \\ 0 & 14 & -4 & -1 \\ 9 & 6 & -6 & 0\end{array}\right]$ and its inverse is matrix $T^{-1}=\frac{1}{9}\left[\begin{array}{rrrr}0 & -2 & -4 & 5 \\ -3 & -2 & 5 & -4 \\ -3 & -5 & -1 & -10 \\ -30 & -8 & -7 & -16\end{array}\right]$. The system (4.1) can be transformed to equivalent system $D(\vec{z})=C \vec{z}+\vec{\nu}$, where 
$\vec{z}=\left[\begin{array}{llll}z_{1} & z_{2} & z_{3} & z_{4}\end{array}\right]^{T}=T^{-1} \vec{x}$ and $\vec{\nu}=T^{-1} \vec{\varphi}$, i.e., we have

$$
\begin{aligned}
& D\left(z_{1}\right)=z_{1} \\
& D\left(z_{2}\right)=z_{3}-6 e^{t} \\
& D\left(z_{3}\right)=z_{4}-6 e^{t} \\
& D\left(z_{4}\right)=-8 z_{2}+6 z_{3}+3 z_{4}-60 e^{t} .
\end{aligned}
$$

Previous system can be transformed into equivalent partially reduced system

$$
\begin{aligned}
D\left(z_{1}\right)-z_{1} & =0 \\
D^{3}\left(z_{2}\right)-3 D^{2}\left(z_{2}\right)-6 D\left(z_{2}\right)+8 z_{2} & =0 \\
z_{3} & =D\left(z_{2}\right)+6 e^{t} \\
z_{4} & =D\left(z_{3}\right)+6 e^{t} .
\end{aligned}
$$

Solution of the previous system is

$$
\left[\begin{array}{l}
z_{1} \\
z_{2} \\
z_{3} \\
z_{4}
\end{array}\right]=\left[\begin{array}{l}
C_{1} e^{t} \\
C_{2} e^{t}+C_{3} e^{-2 t}+C_{4} e^{4 t} \\
C_{2} e^{t}-2 C_{3} e^{-2 t}+4 C_{4} e^{4 t}+6 e^{t} \\
C_{2} e^{t}+4 C_{3} e^{-2 t}+16 C_{4} e^{4 t}+12 e^{t}
\end{array}\right] .
$$

and the solution of the system (4.1) is

$$
\left[\begin{array}{l}
x_{1} \\
x_{2} \\
x_{3} \\
x_{4}
\end{array}\right]=\left[\begin{array}{l}
-3 C_{3} e^{-2 t}-3 C_{4} e^{4 t} \\
-2\left(C_{1}+C_{2}+1\right) e^{t}+C_{3} e^{-2 t}-C_{4} e^{4 t} \\
\left(C_{2}-4\right) e^{t}+2 C_{3} e^{-2 t}-2 C_{4} e^{4 t} \\
\left(C_{1}-4\right) e^{t}+2 C_{3} e^{-2 t}-2 C_{4} e^{4 t}
\end{array}\right] .
$$

By Theorem 3.1 system (4.1) can be transformed into the system

$$
\begin{aligned}
D^{4}\left(x_{1}\right)-4 D^{3}\left(x_{1}\right)-3 D^{2}\left(x_{1}\right)+14 D\left(x_{1}\right)-8 x_{1} & =0 \\
D\left(x_{2}\right)-x_{2} & =x_{1} \\
D\left(x_{3}\right)-x_{3} & =2 x_{1} \\
D\left(x_{4}\right)-x_{4} & =2 x_{1} .
\end{aligned}
$$

Solution of the reduced system is

$$
\left[\begin{array}{l}
x_{1} \\
x_{2} \\
x_{3} \\
x_{4}
\end{array}\right]=\left[\begin{array}{l}
C_{1} e^{t}+C_{2} t e^{t}+C_{3} e^{-2 t}+C_{4} e^{4 t} \\
C_{5} e^{t}+C_{1} e^{t}+\frac{C_{2}}{2} t e^{t}-\frac{C_{3}}{3} e^{-2 t}+\frac{C_{4}}{3} e^{4 t} \\
C_{6} e^{t}+2 C_{1} e^{t}+C_{2} t e^{t}-\frac{2 C_{3}}{3} e^{-2 t}+\frac{2 C_{4}}{3} e^{4 t} \\
C_{7} e^{t}+2 C_{1} e^{t}+C_{2} t e^{t}-\frac{2 C_{3}}{3} e^{-2 t}+\frac{2 C_{4}}{3} e^{4 t}
\end{array}\right] .
$$


To obtain the solution of the system (4.1) we need to find connection between constants $C_{i}, 1 \leq i \leq 7$. Substituting (4.2) into (4.1) we obtain

$$
\begin{aligned}
& \left(C_{1}+C_{2}\right) e^{t}+C_{2} t e^{t}-2 C_{3} e^{-2 t}+4 C_{4} e^{4 t}= \\
& C_{1} e^{t}+C_{2} t e^{t}+C_{3} e^{-2 t}+C_{4} e^{4 t}+\left(C_{1}+C_{5}\right) e^{t}+\frac{C_{2}}{2} t e^{t}-\frac{C_{3}}{3} e^{-2 t}+\frac{C_{4}}{3} e^{4 t}+ \\
& \left(4 C_{1}+2 C_{6}\right) e^{t}+2 C_{2} t e^{t}-\frac{4 C_{3}}{3} e^{-2 t}+\frac{4 C_{4}}{3} e^{4 t}+ \\
& \left(4 C_{1}+2 C_{7}\right) e^{t}+2 C_{2} t e^{t}-\frac{4 C_{3}}{3} e^{-2 t}+\frac{4 C_{4}}{3} e^{4 t}+18 e^{t} \\
& \left(C_{1}+C_{5}+\frac{C_{2}}{2}\right) e^{t}+\frac{C_{2}}{2} t e^{t}+\frac{2 C_{3}}{3} e^{-2 t}+\frac{4 C_{4}}{3} e^{4 t}= \\
& \left(C_{1}+C_{5}\right) e^{t}+\frac{C_{2}}{2} t e^{t}-\frac{C_{3}}{3} e^{-2 t}+\frac{C_{4}}{3} e^{4 t}+C_{1} e^{t}+C_{2} t e^{t}+C_{3} e^{-2 t}+C_{4} e^{4 t} \\
& \left(2 C_{1}+C_{2}+C_{6}\right) e^{t}+C_{2} t e^{t}+\frac{4 C_{3}}{3} e^{-2 t}+\frac{8 C_{4}}{3} e^{4 t}= \\
& \left(2 C_{1}+C_{6}\right) e^{t}+C_{2} t e^{t}-\frac{2 C_{3}}{3} e^{-2 t}+\frac{2 C_{4}}{3} e^{4 t}+2 C_{1} e^{t}+2 C_{2} t e^{t}+2 C_{3} e^{-2 t}+2 C_{4} e^{4 t} \\
& \left(2 C_{1}+C_{2}+C_{7}\right) e^{t}+C_{2} t e^{t}+\frac{4 C_{3}}{3} e^{-2 t}+\frac{8 C_{4}}{3} e^{4 t}= \\
& \left(2 C_{1}+C_{7}\right) e^{t}+C_{2} t e^{t}-\frac{2 C_{3}}{3} e^{-2 t}+\frac{2 C_{4}}{3} e^{4 t}+2 C_{1} e^{t}+2 C_{2} t e^{t}+2 C_{3} e^{-2 t}+2 C_{4} e^{4 t} .
\end{aligned}
$$

Comparing both sides of the equalities, we have $C_{1}=0, C_{2}=0$ and $C_{2}=$ $9 C_{1}+C_{5}+2 C_{6}+2 C_{7}+18$, i.e., we get the solution of the system (4.1)

$$
\left[\begin{array}{l}
x_{1} \\
x_{2} \\
x_{3} \\
x_{4}
\end{array}\right]=\left[\begin{array}{l}
C_{3} e^{-2 t}+C_{4} e^{4 t} \\
-2\left(C_{6}+C_{7}+9\right) e^{t}-\frac{C_{3}}{3} e^{-2 t}+\frac{C_{4}}{3} e^{4 t} \\
C_{6} e^{t}-\frac{2 C_{3}}{3} e^{-2 t}+\frac{2 C_{4}}{3} e^{4 t} \\
C_{7} e^{t}-\frac{2 C_{3}}{3} e^{-2 t}+\frac{2 C_{4}}{3} e^{4 t}
\end{array}\right]
$$

From now on, we will focus on the total reduction method. We will start with calculation of the coefficients of the matrix $\operatorname{adj}(\lambda \mathrm{I}-\mathrm{B}): B_{0}=I, B_{1}=$ $B+d_{1} I=\left[\begin{array}{cccc}-3 & 1 & 2 & 2 \\ 1 & -3 & 0 & 0 \\ 2 & 0 & -3 & 0 \\ 2 & 0 & 0 & -3\end{array}\right], B_{2}=B \cdot B_{1}+d_{2} I=\left[\begin{array}{cccc}3 & -2 & -4 & -4 \\ -2 & -5 & 2 & 2 \\ -4 & 2 & -2 & 4 \\ -4 & 2 & 4 & -2\end{array}\right]$ and $B_{3}=B \cdot B_{2}+d_{3} I=\left[\begin{array}{cccc}-1 & 1 & 2 & 2 \\ 1 & 7 & -2 & -2 \\ 2 & -2 & 4 & -4 \\ 2 & -2 & -4 & 4\end{array}\right]$. Totally reduced system obtained from the system (4.1) is completely decoupled homogenous system of four fourth 
order differential equations which differ only in variables

$$
\begin{aligned}
& D^{4}\left(x_{1}\right)-4 D^{3}\left(x_{1}\right)-3 D^{2}\left(x_{1}\right)+14 D\left(x_{1}\right)-8 x_{1}=0 \\
& D^{4}\left(x_{2}\right)-4 D^{3}\left(x_{2}\right)-3 D^{2}\left(x_{2}\right)+14 D\left(x_{2}\right)-8 x_{2}=0 \\
& D^{4}\left(x_{3}\right)-4 D^{3}\left(x_{3}\right)-3 D^{2}\left(x_{3}\right)+14 D\left(x_{3}\right)-8 x_{3}=0 \\
& D^{4}\left(x_{4}\right)-4 D^{3}\left(x_{4}\right)-3 D^{2}\left(x_{4}\right)+14 D\left(x_{4}\right)-8 x_{4}=0 .
\end{aligned}
$$

Solution of the totally reduced system is

$$
\left[\begin{array}{l}
x_{1} \\
x_{2} \\
x_{3} \\
x_{4}
\end{array}\right]=\left[\begin{array}{l}
C_{1} e^{t}+C_{2} t e^{t}+C_{3} e^{-2 t}+C_{4} e^{4 t} \\
C_{5} e^{t}+C_{6} t e^{t}+C_{7} e^{-2 t}+C_{8} e^{4 t} \\
C_{9} e^{t}+C_{10} t e^{t}+C_{11} e^{-2 t}+C_{12} e^{4 t} \\
C_{13} e^{t}+C_{14} t e^{t}+C_{15} e^{-2 t}+C_{16} e^{4 t}
\end{array}\right] .
$$

Our last task is to find relations between constants $C_{i}$ for $1 \leq i \leq 16$. As we have seen in the previous consideration, we can do that by plugging the solution of the totally reduced system into the original system (4.1). We obtain

$$
\begin{aligned}
& \left(C_{1}+C_{2}\right) e^{t}+C_{2} t e^{t}-2 C_{3} e^{-2 t}+4 C_{4} e^{4 t}= \\
& C_{1} e^{t}+C_{2} t e^{t}+C_{3} e^{-2 t}+C_{4} e^{4 t}+C_{5} e^{t}+C_{6} t e^{t}+C_{7} e^{-2 t}+C_{8} e^{4 t}+ \\
& 2 C_{9} e^{t}+2 C_{10} t e^{t}+2 C_{11} e^{-2 t}+2 C_{12} e^{4 t}+ \\
& 2 C_{13} e^{t}+2 C_{14} t e^{t}+2 C_{15} e^{-2 t}+2 C_{16} e^{4 t}+18 e^{t} \\
& \left(C_{5}+C_{6}\right) e^{t}+C_{6} t e^{t}-2 C_{7} e^{-2 t}+4 C_{8} e^{4 t}= \\
& C_{1} e^{t}+C_{2} t e^{t}+C_{3} e^{-2 t}+C_{4} e^{4 t}+C_{5} e^{t}+C_{6} t e^{t}+C_{7} e^{-2 t}+C_{8} e^{4 t} \\
& \left(C_{9}+C_{10}\right) e^{t}+C_{10} t e^{t}-2 C_{11} e^{-2 t}+4 C_{12} e^{4 t}= \\
& 2 C_{1} e^{t}+2 C_{2} t e^{t}+2 C_{3} e^{-2 t}+2 C_{4} e^{4 t}+C_{9} e^{t}+C_{10} t e^{t}+C_{11} e^{-2 t}+C_{12} e^{4 t} \\
& \left(C_{13}+C_{14}\right) e^{t}+C_{14} t e^{t}-2 C_{15} e^{-2 t}+4 C_{16} e^{4 t}= \\
& 2 C_{1} e^{t}+2 C_{2} t e^{t}+2 C_{3} e^{-2 t}+2 C_{4} e^{4 t}+C_{13} e^{t}+C_{14} t e^{t}+C_{15} e^{-2 t}+C_{16} e^{4 t} .
\end{aligned}
$$

Combining like terms for each equation yields

$$
\begin{aligned}
& C_{2}-C_{1}=C_{5}+2 C_{9}+2 C_{13}+18 \quad 0=C_{6}+2 C_{10}+2 C_{14} \\
& -3 C_{3}=C_{7}+2 C_{11}+2 C_{15} \quad 3 C_{4}=C_{8}+2 C_{12}+2 C_{16} \\
& C_{6}=C_{1} \quad C_{2}=0 \quad-3 C_{7}=C_{3} \quad 3 C_{8}=C_{4} \\
& C_{10}=2 C_{1} \quad C_{2}=0 \quad-3 C_{11}=2 C_{3} \quad 3 C_{12}=2 C_{4} \\
& C_{14}=2 C_{1} \quad C_{2}=0 \quad-3 C_{15}=2 C_{3} \quad 3 C_{16}=2 C_{4} \text {. }
\end{aligned}
$$


By substituting $C_{6}=C_{1}, C_{10}=2 C_{1}$ and $C_{14}=2 C_{1}$ into $C_{6}+2 C_{10}+2 C_{14}=$ 0 , we obtain that $C_{1}=0$. Together with $C_{2}=0$ the first equation becomes $C_{5}+2 C_{9}+2 C_{13}+18=0$. The equation $-3 C_{3}=C_{7}+2 C_{11}+2 C_{15}$ is direct consequence of equations $-3 C_{7}=C_{3},-3 C_{11}=2 C_{3}$ and $-3 C_{15}=2 C_{3}$. Same holds for equations $3 C_{4}=C_{8}+2 C_{12}+2 C_{16}, 3 C_{8}=C_{4}, 3 C_{12}=2 C_{4}$ and $3 C_{16}=2 C_{4}$. Therefore, we get $C_{1}=C_{2}=C_{6}=C_{10}=C_{14}=0, C_{5}=-2\left(C_{9}+C_{13}+9\right)$, $C_{7}=-\frac{C_{3}}{3}, C_{11}=C_{15}=-\frac{2 C_{3}}{3}, C_{8}=\frac{C_{4}}{3}$ and $C_{12}=C_{16}=\frac{2 C_{4}}{3}$. Hence, solution of the system (4.1) is

$$
\left[\begin{array}{l}
x_{1} \\
x_{2} \\
x_{3} \\
x_{4}
\end{array}\right]=\left[\begin{array}{l}
C_{3} e^{-2 t}+C_{4} e^{4 t} \\
-2\left(C_{9}+C_{13}+9\right) e^{t}-\frac{C_{3}}{3} e^{-2 t}+\frac{C_{4}}{3} e^{4 t} \\
C_{9} e^{t}-\frac{2 C_{3}}{3} e^{-2 t}+\frac{2 C_{4}}{3} e^{4 t} \\
C_{13} e^{t}-\frac{2 C_{3}}{3} e^{-2 t}+\frac{2 C_{4}}{3} e^{4 t}
\end{array}\right] .
$$

\section{Acknowledgements}

The authors were supported in part by the Serbian Ministry of Science, Grant No.174032.

\section{RE F E R E N C E S}

1. M. Bixon, J. Jortner: Intramolecular radiationless transitions. J. Chem. Phys. 48 (1968), pp. 715-726.

2. F. Diele, N. Mastronardi, M. Van Barel, E. Van Camp: On computing the spectral decomposition of symmetric arrowhead matrices. Computational Science and Its Applications - ICCSA 2004, 3044 (2004), pp. 932-941.

3. J.W. Gadzuk: Localized vibrational modes in Fermi liquids, general theory. Phys. Rev. B24 (1981), pp. 1651-1663.

4. G.A. Gravvanis: An approximate inverse matrix technique for arrowhead matrices. Int. J. Comput. Math. 70 (1998), pp. 35-45.

5. G.A. Gravvanis: Solving symmetric arrowhead and special tridiagonal linear systems by fast approximate inverse preconditioning. J. Math. Model. Algorithms Oper. Res. 1 (2002), pp. 269-282.

6. M. Gu, S.C. EISEnstat: A divide-and-conquer algorithm for the symmetric tridiagonal eigenproblem. SIAM J. Matrix Anal. Appl. 16 (1995), pp. 172-191.

7. W. HoŁubowski, D. Kurzyk, T. Trawínski: A Fast method for computing the inverse of symmetric block arrowhead matrices. Appl. Math. Inf. Sci. 9 (2015), pp. 319-324.

8. S. VAN HUfFEl, H. PARK: Efficient reduction algorithms for bordered band matrices. Numer. Linear Algebra Appl. 2 (1995), pp 95-113. 
9. N. JakovČević Stor, I. Slapničar, J.L. BARlow: Accurate eigenvalue decomposition of real symmetric arrowhead matrices and applications. Linear Algebra Appl. 464 (2015), pp. 62-89.

10. I. Jovović: Formulae of reduction for some systems of operator equations. Proceedings of International Conference Mathematical and Informational Technologies, MIT-2011, 2011., pp. 161-165.

11. I. Jovović: Total reduction of linear systems of operator equations with the system matrix in the companion form. Publ. Inst. Math. (Beograd) (N.S.) 93 (2013), pp. $117-126$.

12. I. Jovović: Partial reduction for linear systems of operator equations with system matrix in companion form. Novi Sad J. Math. 45 (2015), pp. 1-9.

13. I. Jovović: Differential transcendence of solutions of systems of linear differential equations based on total reduction of the system. Appl. Anal. Discrete Math. 14 (2020), pp. 498-511.

14. H.T. Kung, B.W. Suter: A hub matrix theory and applications to wireless communications. EURASIP J. Adv. Signal Process. 2007 (2007), 013659.

15. Z. LIU, K. WANG, C. XU: Extremal inverse eigenvalue problem for symmetric doubly arrow matrices. J. Appl. Math. Comput. 45 (2014), pp. 151-164.

16. B. Malešević, D. Todorić, I. Jovović, S. Telebaković: Formulae of partial reduction for linear systems of first order operator equations. Appl. Math. Lett. 23 (2010), pp. 1367-1371.

17. B. Malešević, D. Todorić, I. Jovović, S. Telebaković: Differential transcendency in the theory of linear differential systems with constant coefficients. ISRN Math. Anal. 2012 (2012), pp. 1-8.

18. E. Mizutani, J.W. Demmel: On structure-exploiting trust-region regularized nonlinear least squares algorithms for neural-network learning. Neural Networks, 16 (2003), pp. 745-753.

19. D. Mogilevtsev, A. Maloshtan, S. Kilin, L.E. Oliveira, S.B. Cavalcanti: Spontaneous emission and qubit transfer in spin-1/2 chains. J. Phys. B43 (2010), 095506.

20. E. Montaño, M. Salas, R.L. Soto: Positive matrices with prescribed singular values. Proyecciones, 27 (2008), pp. 289-305.

21. D.P. O'Leary, G.W. Stewart: Computing the eigenvalues and eigenvectors of symmetric arrowhead matrices. J. Comput. Phys. 90 (1990), pp 497-505.

22. S. OliveIRA: A new parallel chasing algorithm for transforming arrowhead matrices to tridiagonal form. Math. Comp. 67 (1998), pp. 221-235.

23. B.N. PARlett, B. Nour-Omid: The use of refined error bound when updating eigenvalues of tridiagonals. Linear Algebra Appl. 68 (1985), pp. 179-219.

24. J. Peng, X.Y. Hu, L. ZhANG: Two inverse eigenvalue problems for a special kind of matrices. Linear Algebra Appl. 416 (2006), pp. 336-347.

25. H. Pickmann, J. Egana, R.L. Soto: Extremal inverse eigenvalue problem for bordered diagonal matrices. Linear Algebra Appl. 427 (2007), pp. 256-271.

26. H. Saberi Najafi, S.A. Edalatpanah, G.A. Gravvanis: An efficient method for computing the inverse of arrowhead matrices. Appl. Math. Lett. 33 (2014), pp. $1-5$. 
27. L. SHen, B.W. Suter: Bounds for eigenvalues of arrowhead matrices and their applications to hub matrices and wireless communications. EURASIP J. Adv. Signal Process. 2009 (2009), 379402.

28. Y. YUAN: Generalized inverse eigenvalue problems for symmetric arrowhead matrices. Int. J. Math. Comput. Sci. 4 (2010), pp. 905 - 908.

29. H. ZHA: A two-way chasing scheme for reducing a symmetric arrowhead matrix to tridiagonal form. J. Numer. Linear Algebra Appl. 1 (1992), pp. 49-57. 